\title{
Frequency-comb-referenced molecular spectroscopy in the mid-infrared region
}

\section{Vainio, Markku}

2011

Vainio , M , Merimaa , M \& Halonen , L 2011 , ' Frequency-comb-referenced molecular spectroscopy in the mid-infrared region ' , Optics Letters , vol. 36 , no. 21 , pp. 4122-4144 . https://doi.org/10.1364/O

http://hdl.handle.net/10138/28130

https://doi.org/10.1364/OL.36.004122

acceptedVersion

Downloaded from Helda, University of Helsinki institutional repository.

This is an electronic reprint of the original article.

This reprint may differ from the original in pagination and typographic detail.

Please cite the original version. 


\title{
Frequency-comb-referenced molecular spectroscopy in the mid-infrared region
}

\author{
Markku Vainio, ${ }^{1,2, *}$ Mikko Merimaa, ${ }^{1}$ and Lauri Halonen ${ }^{2}$ \\ ${ }^{1}$ Centre for Metrology and Accreditation (MIKES), P. O. Box 9, FIN-02151 Espoo, Finland \\ ${ }^{2}$ Laboratory of Physical Chemistry, Department of Chemistry, P. O. Box 55, (A. I. Virtasen aukio 1), \\ FIN-00014 University of Helsinki, Finland \\ ${ }^{*}$ Corresponding author: markku.vainio@helsinki.fi
}

Received July 28, 2011; revised September 20, 2011; accepted September 20, 2011; posted September 20, 2011 (Doc. ID 151946); published October 19, 2011

\begin{abstract}
A simple method for absolute-frequency measurements of molecular transitions in the mid-IR region is reported. The method is based on a cw singly resonant optical parametric oscillator (SRO), which is tunable from 3.2 to $3.45 \mu \mathrm{m}$. The mid-IR frequency of the SRO is referenced to an optical frequency comb through its pump and signal beams. Sub-Doppler spectroscopy and absolute-frequency measurement of the $P(7)$ transition of the $\nu_{3}$ band of $\mathrm{CH}_{4}$ are demonstrated. (c) 2011 Optical Society of America OCIS codes: $\quad 120.6200,190.4970,300.6320,300.6340$.
\end{abstract}

Optical frequency combs (OFCs) based on mode-locked fs lasers can be used to phase-coherently link the visible and near-IR parts of the optical spectrum to microwave frequencies, and therefore to the SI second. Establishing a similar link between the mid-IR and microwaves is more challenging owing to the lack of mode-locked mid-IR lasers that could support an octave-spanning spectrum, which is a prerequisite to self-referenced operation of an OFC. The most promising technique for mid-IR OFC generation is based on synchronously pumped optical parametric oscillators (OPOs) [1-3], in particular to those operated at degeneracy $[1,2]$. In another approach, the need for self-referenced operation in the mid-IR is circumvented by transferring a visible or near-IR OFC to the mid-IR by optical difference frequency mixing $[\underline{4}, 5]$.

In addition to direct and indirect generation of mid-IR OFCs, absolute-frequency measurement techniques based on cw lasers have been developed. The basic idea common to all such techniques is to use nonlinear optics to link a cw mid-IR beam to a visible/near-IR OFC and hence further to a microwave frequency standard. This can be done by difference frequency generation [6,7], sum-frequency mixing [8], or by using a cw optical parametric oscillator that is pumped with a near-IR laser [9]. These techniques have been used to measure absolute frequencies of some molecular transitions between 3 and $5 \mu \mathrm{m}$ [6-8]. This wavelength region contains fundamental vibrational bands of $\mathrm{CH}, \mathrm{NH}$, and $\mathrm{OH}$ stretching modes, and is particularly important in molecular spectroscopy.

In this Letter, we present a new scheme for mid-IR absolute-frequency spectroscopy. The basic idea is similar to that previously reported by Kovalchuk et al. for frequency comparison between a $3.39 \mu \mathrm{m} \mathrm{CH}_{4}$-stabilized HeNe laser and a $532 \mathrm{~nm} \mathrm{I}_{2} / \mathrm{Nd}$ :YAG laser [9]. Kovalchuk et al. used non-phase-matched components of a pumpresonant, singly resonant OPO [10] to bridge the two wavelength regions [9]. Our solution relies on the use of a cw optical parametric oscillator that is truly singly resonant (SRO). The SRO produces a tunable, high-power mid-IR beam that can be directly used in sub-Doppler molecular spectroscopy while being referenced to a visible or near-IR OFC in a simple manner. In the following, we describe the method and its realization.

An SRO is pumped with a laser at frequency $\nu_{p}$, and it produces two output beams. These output beams are called the signal and idler, and their frequencies $\left(\nu_{s}\right.$ and $\nu_{i}$, respectively) are linked to the pump frequency by the law of energy conservation:

$$
\nu_{p}=\nu_{s}+\nu_{i}
$$

The wavelength $\lambda_{i}$ of the idler of a typical mid-IR cw SRO is widely tunable at $3-4 \mu \mathrm{m}$. In the most common implementation, the pump beam is produced by a high-power $\mathrm{cw}$ laser operating at $\lambda_{p} \approx 1 \mu \mathrm{m}$. As a result, the signal wavelength $\lambda_{s}$ is approximately $1.5 \mu \mathrm{m}$.

According to Eq. (1), the frequency of the mid-IR idler beam can be unambiguously determined by measuring the frequencies of the pump and signal beams. This can most easily be done using an OFC generator based on an Er-doped fiber laser, in which case the pump and signal wavelengths are directly within the spectral coverage $(1.0$ to $2.1 \mu \mathrm{m})$ of the OFC. Another solution, which is the one we have used in this work, is to frequency double the pump and signal beams, so that their frequencies can be measured using a Ti:sapphire comb that typically extends from 0.5 to $1.1 \mu \mathrm{m}$.

An OFC based on a mode-locked laser consists of a large number of equidistant laser peaks, and the optical frequency of the $m$ th peak is $f_{m}=f_{\text {ceo }}+m f_{\text {rep }}$, where $f_{\text {ceo }}$ is the carrier-envelope offset frequency and $f_{\text {rep }}$ is the repetition rate of the comb laser. When the frequencies of the SRO's pump and signal are measured against the adjacent comb peaks $m_{p}$ and $m_{s}$, respectively, beat frequencies $f_{p}=f_{m(p)}-\nu_{p}$ and $f_{s}=f_{m(s)}-\nu_{s}$ are observed. The absolute frequency of the idler beam can then be obtained, using Eq. (1), as $\nu_{i}=\left(m_{p}-m_{s}\right) f_{\text {rep }} \pm$ $f_{s} \pm f_{p}$. (Or, $\nu_{i}=1 / 2\left[\left(m_{2 p}-m_{2 s}\right) f_{\text {rep }} \pm f_{2 s} \pm f_{2 p}\right]$ if the frequency-doubled pump and signal beams are referenced to the comb). The repetition rate of the comb laser is typically locked to a microwave frequency standard (such as a Cs-clock or a hydrogen maser) and is known with high accuracy. The comb mode numbers, $m_{p}$ and 
$m_{s}$, can be obtained by measuring frequencies $\nu_{p}$ and $\nu_{s}$ coarsely with an accurate wavelength meter. Beat frequencies $f_{p}$ and $f_{s}$ can be counted simultaneously with two frequency counters referenced to a frequency standard. We point out that the measurement is independent

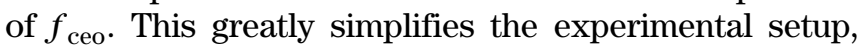
since $f_{\text {ceo }}$ does not have to be stabilized or measured.

The experimental realization of the method is schematically shown in Fig. 1. The SRO is essentially similar to the one described in our earlier work [11]. The SRO cavity is a bow-tie ring cavity, which comprises four mirrors that are all highly reflective $(>99.9 \%)$ at the signal wavelength. The pump laser system outputs up to $15 \mathrm{~W}$ of linearly polarized light at $1064 \mathrm{~nm}$. The nonlinear crystal is a $5 \mathrm{~cm}$ long periodically poled $5 \% \mathrm{MgO}$-doped lithium niobate (MgO:PPLN). Coarse tuning of the idler wavelength from 3.2 to $3.45 \mu \mathrm{m}$ is achieved with a single poling period $(30.5 \mu \mathrm{m})$ by varying the MgO:PPLN temperature. Mode-hop-free fine tuning of the idler over more than $90 \mathrm{GHz}$ is obtained at any set point by tuning the pump laser with a piezoelectric actuator (PZT). In addition, the signal frequency can be fine tuned by up to one cavity free spectral range $(580 \mathrm{MHz})$ by changing the length of the SRO cavity with another PZT (see Fig. 1). The maximum single-mode output power of the SRO idler beam is $>0.5 \mathrm{~W}$.

The OFC generator used in this work has been described in detail in previous publications $[12,13]$. It is based on a mode-locked fs Ti:sapphire laser. The repetition rate of the laser is approximately $1 \mathrm{GHz}$, and it is locked to an active hydrogen maser, which is traceable to the SI-second through UTC. In order to reference the SRO pump frequency to the Ti:sapphire OFC, a fraction of the pump laser beam is picked before the SRO and frequency doubled in a $3 \mathrm{~mm}$ long potassium titanyl phosphate crystal. Single-pass doubling with type II (eo-e) phase matching is used to produce more than $1 \mathrm{~mW}$ of green $(532 \mathrm{~nm}$ ) with a pump power of $2 \mathrm{~W}$. A single-mode optical fiber is used to transfer the green light to another optical table that accommodates the OFC. The light exiting the fiber is overlapped with the green part of the OFC spectrum in a nonpolarizing beam splitter, and a grating filters out the unwanted spectral components before the detection of beat signal $f_{2 p}$ with a fast photodiode. The signal-to-noise ratio (SNR) of $f_{2 p}$ is $30-40 \mathrm{~dB}$ in a resolution bandwidth (RBW) of $300 \mathrm{kHz}$.

Generation of $2 \nu_{s}$ does not require a separate frequency doubler, since a significant amount of stable

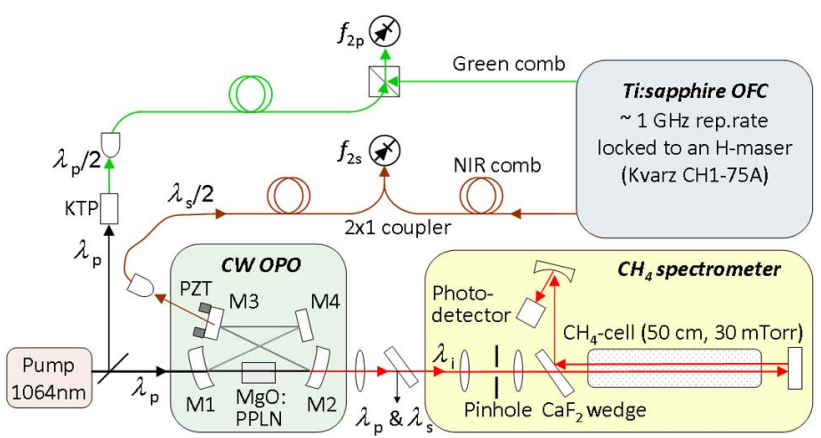

Fig. 1. (Color online) Simplified schematic of the experimental setup. second harmonic power of the signal is produced in the MgO:PPLN of the SRO as a result of a residual nonlinear mixing process. Depending on the wavelength of the signal beam, and with $>0.3 \mathrm{~W}$ of idler output power, the frequency-doubled power exiting the SRO through mirror M3 (Fig. 1) is 50 to $800 \mu \mathrm{W}$, as measured after a gold-coated grating that separates $\lambda_{s} / 2$ from other wavelengths emitted by the SRO. The $\lambda_{s} / 2$ beam is coupled into a single-mode fiber and combined with the near-IR part of the OFC in a $2 \times 1$ fiber coupler. The two overlapping beams are filtered with a grating and passed to a fast photodiode for beat-frequency measurement. The SNR of this beat signal $\left(f_{2 s}\right)$ is 15 to $25 \mathrm{~dB}$ in a $300 \mathrm{kHz}$ RBW, which is sufficient for frequency counting after band-pass filtering. Much higher output power at $2 \nu_{s}$, and hence a better SNR of $f_{2 s}$ could be obtained by using an additional frequency-doubling crystal inside the SRO cavity [14].

As the first test of the method and instrumentation, we measured the frequency stability of the free-running SRO. The recorded beat frequencies, as well as the idler frequency deviation calculated from them, are shown in Fig. 2. The corresponding Allan deviation is plotted in Fig. 3. This measurement allows frequency drifts of the SRO to be studied with much higher precision than what is possible with a wavelength meter [11], revealing the exceptional stability of our SRO. Similar stability has previously been reported only for actively stabilized SROs $[14,15]$ and/or for pump-resonant SROs [10].

The good passive stability and high output power of the SRO allow sub-Doppler spectroscopy to be done without a power enhancement cavity. A simple saturatedabsorption spectrometer outlined in Fig. 1 is sufficient. An example of a sub-Doppler spectrum of methane measured with the setup is shown in Fig. 4. The single-pass mid-IR power in the sample cell was $600 \mathrm{~mW}$, and the $1 / e^{2}$ diameter of the collimated mid-IR beam was $2.7 \mathrm{~mm}$. This corresponds to an order of magnitude larger intensity than the saturation intensity. The spectrum was recorded by continuously scanning the SRO frequency over the line and by averaging several scans (over $5 \mathrm{~min}$.) in order to improve the SNR of the measurement. Averaging a large number of scans is made possible by the link

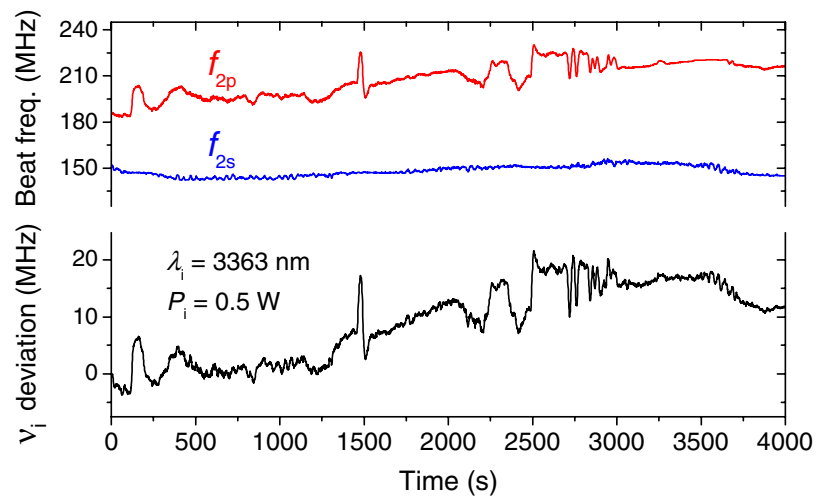

Fig. 2. (Color online) Frequency stability of the free-running SRO. The upper panel shows the individual beat signals, including a contribution from the unstabilized $f_{\text {ceo }}$. The lower panel shows the idler frequency deviation calculated from the beat frequencies, with the effect of $f_{\text {ceo }}$ canceled out. Counter gate time $1 \mathrm{~s}$. 


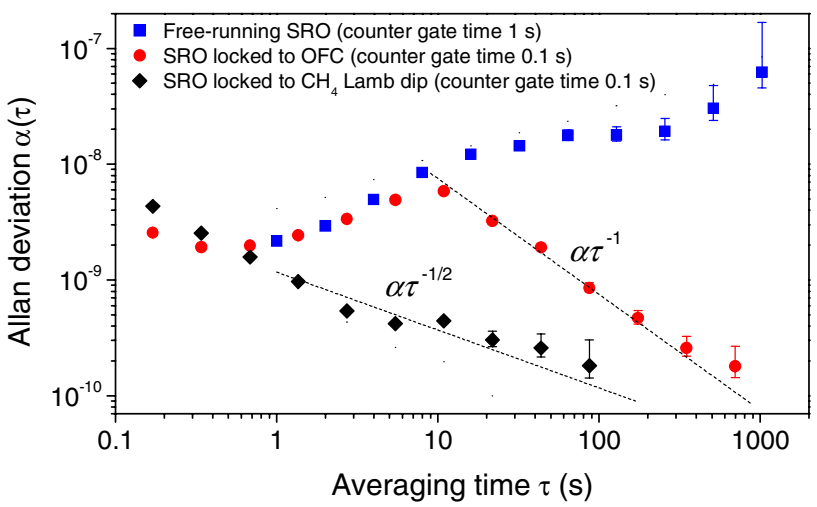

Fig. 3. (Color online) Relative Allan deviation of the freerunning SRO (blue squares) as calculated from the data of Fig. 2. Also shown are the Allan deviations of the $\mathrm{SRO}$ when its frequency is locked to the OFC and to the $F_{2}^{(2)} P(7) \nu_{3}$ transition of ${ }^{12} \mathrm{CH}_{4}$.

to the $\mathrm{OFC}$, which gives the absolute-frequency scale and makes the measurement less sensitive to SRO frequency drifts and intensity noise. The observed linewidth of the Lamb dip is mainly due to SRO frequency jitter $(>2 \mathrm{MHz})$, and due to power broadening [6].

Further improvement of the long-term stability of the SRO was achieved by locking its pump and signal frequencies to the OFC. The beat-frequency counter readings were used as error signals, and a software-based integrator produced the corrections that were fed into the PZT amplifiers of the pump laser and SRO cavity, so as to stabilize the respective frequencies. The Allan deviation of the OFC-locked SRO is shown in Fig. 3. Counter gate time of $0.1 \mathrm{~s}$ was used, and the bandwidth of each feedback loop was $<1 \mathrm{~Hz}$. A tighter lock based on phase-locked loops could be implemented for improved short-term stability and for true single-frequency synthesis [13].

Another way of improving the stability of the SRO idler frequency is to lock it to a molecular absorption line. Figure 3 shows the Allan deviation of the SRO locked to the Lamb dip of the $F_{2}^{(2)} P(7)$ component of $\mathrm{CH}_{4}$ at $3.39 \mu \mathrm{m}$. Wavelength modulation spectroscopy with the standard third harmonic ( $3 f$ ) detection was used to produce the error signal for locking, and an electronic integrator was used to close the servo loop. Both the modulation and the correction signal from the integrator were applied to the PZT of the SRO cavity mirror M3 (Fig. 1). Modulation frequency was $f=1249 \mathrm{~Hz}$ and the modulation depth was $\sim 3.2 \mathrm{MHz}$ peak to peak. An example of the $3 f$ signal is shown in the inset of Fig. $\underline{4}$, together with a theoretical signal calculated from a direct recording of the Lamb dip (thick gray line). The frequency of the line center deduced from the measurement is $88376181355 \mathrm{kHz}$, with a standard deviation of $664 \mathrm{kHz}$ (380 s measurement, $0.1 \mathrm{~s}$ counter gate time). The measured value is $246 \mathrm{kHz}$ smaller than the Comité International des Poids et Mesures recommended value [16]. This deviation is due to the fact that the measurement was not done under the conditions of the mise

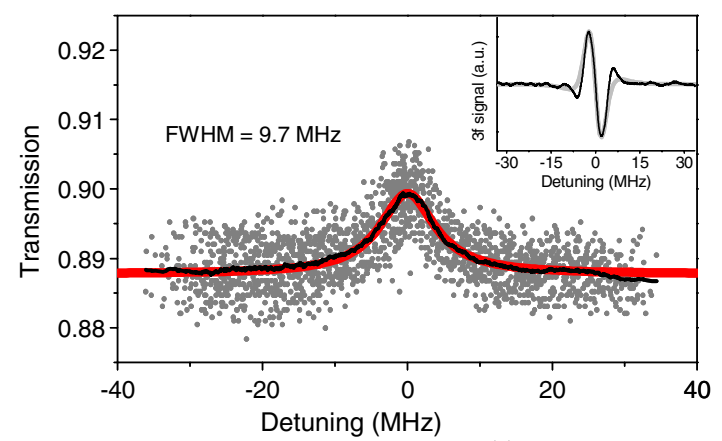

Fig. 4. (Color online) Lamb dip of the $F_{2}^{(2)} P(7) \nu_{3}$ transition of ${ }^{12} \mathrm{CH}_{4}$. Frequency detuning is relative to the line center. Dots are data from several scans over the line, and the black solid line is the average of all scans. The thick red line is a Lorentzian fit to the data. The inset shows a $3 f$ recording of the Lamb dip. Time constant of the lock-in amplifier was $1 \mathrm{~ms}$, and the sweep rate was $440 \mathrm{MHz} / \mathrm{s}$.

en pratique of the definition of the meter [16]. Also, we have not yet characterized the systematic frequency shifts of the spectrometer. Therefore, no corrections were applied to the measured value and no uncertainty for it is given.

We thank the Academy of Finland for financial support.

\section{References}

1. N. Leindecker, A. Marandi, R. L. Byer, and K. L. Vodopyanov, Opt. Express 19, 6296 (2011).

2. S. T. Wong, T. Plettner, K. L. Vodopyanov, K. Urbanek, M. Digonnet, and R. L. Byer, Opt. Lett. 33, 1896 (2008).

3. F. Adler, K. C. Cossel, M. J. Thorpe, I. Hartl, M. E. Fermann, and J. Ye, Opt. Lett. 34, 1330 (2009).

4. S. M. Foreman, A. Marian, J. Ye, E. A. Petrukhin, M. A. Gubin, O. D. Mücke, F. N. C. Wong, E. P. Ippen, and F. X. Kärtner, Opt. Lett. 30, 570 (2005).

5. P. Maddaloni, P. Malara, G. Gagliardi, and P. De Natale, New J. Phys. 8, 262 (2006).

6. D. Mazzotti, P. Cancio, G. Giusfredi, P. De Natale, and M. Prevedelli, Opt. Lett. 30, 997 (2005).

7. K. Takahata, T. Kobayashi, H. Sasada, Y. Nakajima, H. Inaba, and F.-L. Hong, Phys. Rev. A 80, 032518 (2009).

8. S. Borri, S. Bartalini, I. Galli, P. Cancio, G. Giusfredi, D. Mazzotti, A. Castrillo, L. Gianfrani, and P. De Natale, Opt. Express 16, 11637 (2008).

9. E. V. Kovalchuk, T. Schuldt, and A. Peters, Opt. Lett. 30, 3141 (2005).

10. E. V. Kovalchuk, D. Dekorsy, A. I. Lvovsky, C. Braxmaier, J. Mlynek, A. Peters, and S. Schiller, Opt. Lett. 26, 1430 (2001).

11. M. Vainio, J. Peltola, S. Persijn, F. J. M. Harren, and L. Halonen, Opt. Express 16, 11141 (2008).

12. M. Merimaa, K. Nyholm, M. Vainio, and A. Lassila, IEEE Trans. Instrum. Meas. 56, 500 (2007).

13. V. Ahtee, M. Merimaa, and K. Nyholm, Opt. Express 17, 4890 (2009).

14. O. Mhibik, T.-H. My, D. Pabœuf, F. Bretenaker, and C. Drag, Opt. Lett. 35, 2364 (2010).

15. S. Zaske, D.-H. Lee, and C. Becher, Appl. Phys. B 98, 729 (2009).

16. T. J. Quinn, Metrologia 40, 103 (2003). 